\title{
Structural and Ferroic Properties of La, Nd, and Dy Doped $\mathrm{BiFeO}_{3}$ Ceramics
}

\author{
Ashwini Kumar, Poorva Sharma, and Dinesh Varshney \\ Materials Science Laboratory, School of Physics, Vigyan Bhawan, Devi Ahilya University, Khandwa Road Campus, \\ Indore 452001, India
}

Correspondence should be addressed to Ashwini Kumar; ashu1220@gmail.com

Received 20 August 2014; Accepted 24 November 2014

Academic Editor: Shaomin Liu

Copyright (C) 2015 Ashwini Kumar et al. This is an open access article distributed under the Creative Commons Attribution License, which permits unrestricted use, distribution, and reproduction in any medium, provided the original work is properly cited.

Polycrystalline samples of $\mathrm{Bi}_{0.8} R E_{0.2} \mathrm{FeO}_{3}(R E=\mathrm{La}, \mathrm{Nd}$, and $\mathrm{Dy})$ have been synthesized by solid-state reaction route. $\mathrm{X}$ ray diffraction (XRD) patterns of $\mathrm{Bi}_{0.8} \mathrm{La}_{0.2} \mathrm{FeO}_{3}$ and $\mathrm{Bi}_{0.8} \mathrm{Nd}_{0.2} \mathrm{FeO}_{3}$ were indexed in rhombohedral $(R 3 c)$ and triclinic (P1) structure, respectively. Rietveld refined XRD pattern of $\mathrm{Bi}_{0.8} \mathrm{Dy}_{0.2} \mathrm{FeO}_{3}$ confirms the biphasic (Pnma $+R 3 c$ space groups) nature. Raman spectroscopy reveals the change in $\mathrm{BiFeO}_{3}$ mode positions and supplements structural change with $R E$ ion substitution. Ferroelectric and ferromagnetic loops have been observed in the $\mathrm{Bi}_{0.8} R E_{0.2} \mathrm{FeO}_{3}$ ceramics at room temperature, indicating that ferroelectric and ferromagnetic ordering coexist in the ceramics at room temperature. The magnetic measurements at room temperature indicate that rare-earth substitution induces ferromagnetism and discerns large and nonzero remnant magnetization as compared to pristine $\mathrm{BiFeO}_{3}$.

\section{Introduction}

Multiferroic materials, such as $\mathrm{BiFeO}_{3}(\mathrm{BFO})$, have been a subject of unprecedented interest due to coexistence of simultaneous ferroelectric and antiferromagnetic/ferromagnetic ordering in the same phase [1]. These are studied extensively due to their wide range of potential applications, including information-storage device, spintronics, and sensors $[2,3]$. The common exclusive nature of magnetism and electric polarization makes natural multiferroic materials rare $[3,4]$. Needless to say, majority of compounds have low ordering temperatures; however room temperature achievement has yet to be noticed. BFO has a rhombohedrally distorted perovskite structure (space group R3c) [5] with high Curie temperature $\left(T_{C} \sim 1100 \mathrm{~K}\right)$ and antiferromagnetic Neel temperature $\left(T_{N} \sim 675 \mathrm{~K}\right)$ with a spatially modulated spiral spin structure $[6,7]$.

Efforts have been made to improve the ferroelectric and magnetic properties in antiferromagnetic $\mathrm{BiFeO}_{3}$ ceramic. Enhancing the magnetic moments by reducing particle size of $\mathrm{BFO}$ has been one of the important tasks [8]. Ion substitution in $\mathrm{BFO}$ is believed to be effective and the most convenient way to enhance the ferroelectric and magnetic properties.
From the existing literature, it has been earlier observed that partial substitution of rare-earth $(R E)$ elements like La [9], Pr [10], Nd [11, 12], Gd [13], Dy [14], and Ho [15] at $\mathrm{Bi}$ site can eliminate the impurity phase along with a structural phase transformation and improve ferroelectric properties and induced ferromagnetism in BFO ceramic. The relationship between the structural, evolution, and magnetic properties among these doped $\mathrm{BFO}$ ceramics still needs further investigations. With these priorities, we have synthesized $R E$ ions doped (Bi site) BFO ceramics.

A structural phase transition from rhombohedral to orthorhombic structure is observed in $\mathrm{Bi}_{1-x} \mathrm{La}_{x} \mathrm{FeO}_{3}$ near $x=0.3$ and enhances the magnetoelectric interaction [9]. For $\mathrm{Nd}$ doped $\mathrm{BiFeO}_{3}$ a rhombohedral structure at $x=$ 0 , a triclinic structure between $x=0.05$ and 0.15 , and a pseudotetragonal structure between $x=0.175$ and 0.2 have been reported [11]. A structural transformation from rhombohedral to monoclinic structure for $\mathrm{Bi}_{1-x} \mathrm{Nd}_{x} \mathrm{FeO}_{3}(x=$ $0.0-0.15)$ ceramic prepared by an improved rapid liquid phase sintering method is also reported [12]. On the other hand, 20\% Dy substitution confirms the orthorhombic structure with Pnma structural model [14]. Substitutional effect of Ho on $\mathrm{BFO}$ bulk ceramic infers that the remnant polarization 
$\left(P_{r}\right)$ and switching characteristic were improved at low field by reducing the leakage current apart from enhanced ferromagnetic properties [15]. These improved properties obtained by RE doping demonstrate the possibility of enhancing multiferroic applicability of BFO.

From this viewpoint, it is necessary to be aware of the crystal structure of compounds. Keeping the important features in mind and taking interesting crystallography of $\mathrm{BiFeO}_{3}$ into consideration, it is worth studying the properties of rare-earth doped $\mathrm{Bi}$-ferrite. We have thus synthesized rareearth substituted $\mathrm{BiFeO}_{3}$ abbreviated as $\mathrm{Bi}_{0.8} R E_{0.2} \mathrm{FeO}_{3}(R E=$ $\mathrm{La}, \mathrm{Nd}$, and Dy) multiferroic samples via solid-state reaction route and reported the consistency of evolution of crystal structure, dielectric, electrical, and magnetic properties. The synthesized samples of $\mathrm{Bi}_{0.8} R E_{0.2} \mathrm{FeO}_{3}(R E=\mathrm{La}, \mathrm{Nd}$, and Dy) are further designated as BLFO, BNFO, and BDFO, respectively.

\section{Experimental Details}

Polycrystalline samples with the composition $\mathrm{Bi}_{0.8} R E_{0.2} \mathrm{FeO}_{3}$ $(R E=\mathrm{La}, \mathrm{Nd}$, and $\mathrm{Dy})$ were prepared by conventional solid-state route and the typical synthesized process is described as follows. For the synthesis of $\mathrm{Bi}_{0.8} R E_{0.2} \mathrm{FeO}_{3}$ $(R E=\mathrm{La}, \mathrm{Nd}$, and $\mathrm{Dy})$ ceramics $\mathrm{Bi}_{2} \mathrm{O}_{3}$ (Loba Chemie, 99.9\% purity), $\mathrm{Fe}_{2} \mathrm{O}_{3}$ (Sigma-Aldrich, $99.99 \%$ purity), $\mathrm{La}_{2} \mathrm{O}_{3}$ (Sigma-Aldrich, 99.9\% purity), $\mathrm{Nd}_{2} \mathrm{O}_{3}$ (Loba Chemie, 99.9\% purity), and $\mathrm{Dy}_{2} \mathrm{O}_{3}$ (Loba Chemie, 99.9\% purity) reagents were used as starting materials. All the chemicals were of GR grade and were used without any further purification. All the starting materials were weighed, mixed, and grounded thoroughly in an agate mortar for 6 hours using acetone and calcined for 6 hours at $650^{\circ} \mathrm{C}$ for the desired composition of $\mathrm{Bi}_{0.8} R E_{0.2} \mathrm{FeO}_{3}$. All the calcined compositions were uniaxially dye-pressed into pellets of size $10 \mathrm{~mm}$ in diameter and $2 \mathrm{~mm}$ in thickness. Sintering was performed at $820^{\circ} \mathrm{C}$ for 3 hours, with intermediate grinding.

X-ray diffraction was carried out with $\mathrm{CuK} \alpha_{1}(1.5406 \AA)$ radiation using Bruker D8 Advance X-ray diffractometer over the angular range $2 \theta\left(20^{\circ}-80^{\circ}\right)$ with a scanning rate of $2^{\circ}$ per minute at room temperature working at $40 \mathrm{kV}$ voltage and $40 \mathrm{~mA}$ current. The lattice parameters and other detailed structural information were obtained by the Rietveld refinement FullPROF program [16]. Raman measurements were carried out using LabRam HR800 micro-Raman spectrometer equipped with a 50x objective, an appropriate edge filter, and a Peltier-cooled charge coupled device detector. The spectra were excited with $488 \mathrm{~nm}$ radiations $(2.53 \mathrm{eV})$ from an air-cooled Argon laser. Ferroelectric hysteresis $(P$ $E$ ) loop of the samples was measured using RT6000 (Radiant Technologies, USA) at frequency $50 \mathrm{~Hz}$. The magnetization as a function of applied field $(M-H)$ curve for BNFO sample at room temperatures was performed using a superconducting quantum interference device (SQUID) magnetometer (MPMS, Quantum Design). However, for BLFO and BDFO samples magnetic measurements were performed using vibrating sample magnetometer (VSM-lakeshore).

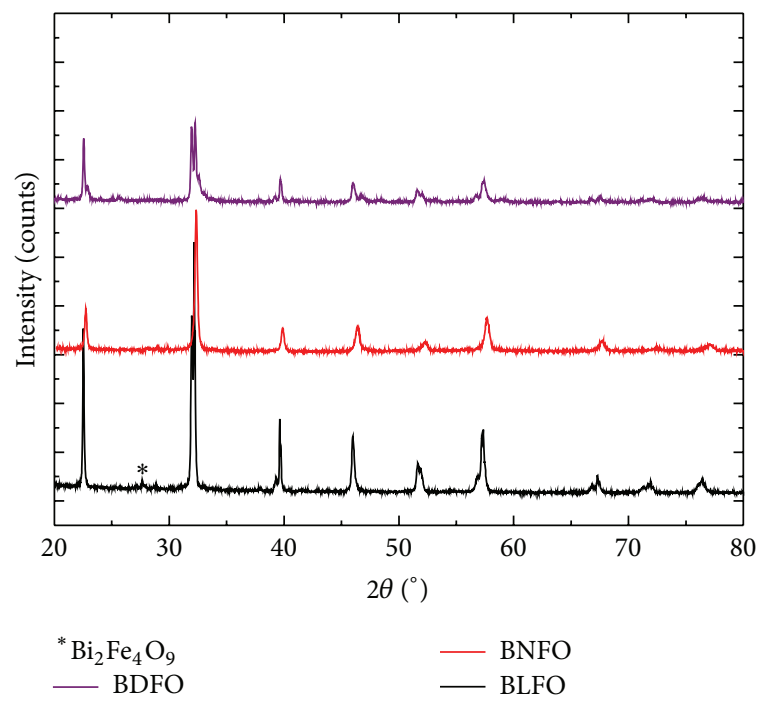

FIgURE 1: Room temperature XRD pattern of the $\mathrm{Bi}_{0.8} R E_{0.2} \mathrm{FeO}_{3}(R E$ $=\mathrm{La}, \mathrm{Nd}$, and Dy) samples designated as BLFO, BNFO, and BDFO, respectively.

\section{Results and Discussion}

3.1. Structural Analysis. Figure 1 shows the XRD pattern of $\mathrm{Bi}_{0.8} R E_{0.2} \mathrm{FeO}_{3}(R E=\mathrm{La}, \mathrm{Nd}$, and $\mathrm{Dy})$ ceramic samples. From the measured XRD pattern it has been observed that all the samples exhibit different crystal structures. BLFO sample was indexed in rhombohedral structure with space group $R 3 c$ where all the diffraction peaks match closely with the JCPDS file number 86-1518. A minor low level impurity phase (marked with $*$ ) was detected around $2 \theta \approx 28^{\circ}$ associated with $\mathrm{Bi}_{2} \mathrm{Fe}_{4} \mathrm{O}_{9}$ [17]. This impurity peak matches well with the JCPDS file number 72-1832. The diffraction peaks change in both intensity and $2 \theta$ values with different dopant ionic radii as a result of change in crystal structure [18]. The values of lattice constants were manually calculated using different forms of crystal structures as defined earlier [19].

In order to further analyze the crystal structure the Rietveld refinement of measured XRD pattern was performed for all the samples as shown in Figures 2(a), 2(b), and 2(c). La doped BFO compound holds the polar rhombohedral $R 3 c$ structure similar to pure bismuth ferrite (BFO). The XRD pattern of BLFO sample was indexed in rhombohedral $(R 3 c)$ system with lattice parameters $a=b=5.5604(5) \AA$ and $c=$ 13.7596(6) $\AA$ as shown in Figure 2(a). Earlier study reports a substitutional induced structural phase transition $(R 3 c \rightarrow$ C222) for BLFO compound $[9,21]$. It is worth noting that our attempt to fit the XRD pattern of present BLFO sample with C222 structural model was completely failed. Indeed, the reflection conditions derived from indexed reflection for BLFO cell were $l=2 n$ for $h h l, k=2 n$ for $h k h, h=2 n$ for $h k k$, $h=2 n$ for $h 00, k=2 n$ for $0 k 0$, and $l=2 n$ for $00 l$ which are compatible with the space group $R 3 c$.

The XRD pattern of BNFO sample was indexed in triclinic structure ( $P 1$ space group) with cell parameters $a=$ 3.9074(5) $\AA, b=3.9112(6) \AA$, and $c=3.9002(5) \AA$. The $\mathrm{X}$-ray diffraction pattern shows that all the X-ray peaks of 


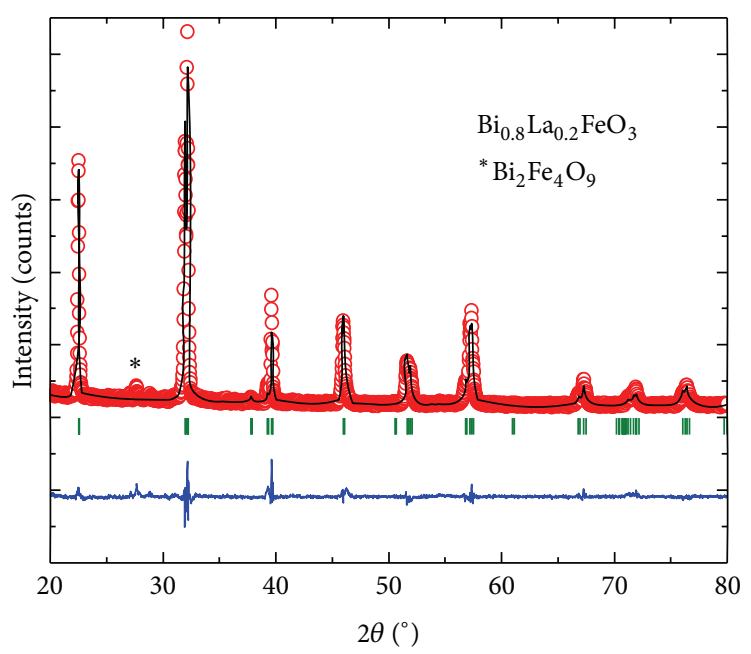

(a)

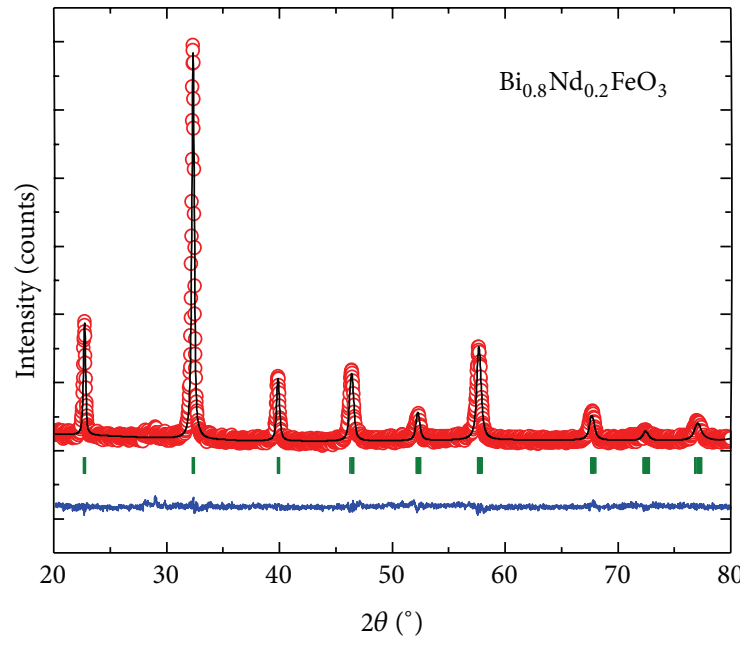

(b)

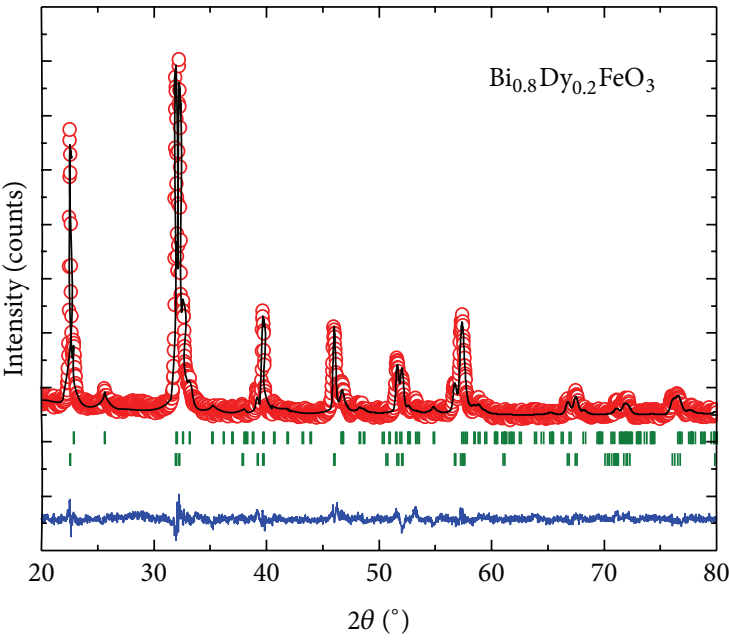

(c)

FIGURE 2: Rietveld refined X-ray diffraction pattern of the $\mathrm{Bi}_{0.8} R E_{0.2} \mathrm{FeO}_{3}(R E=\mathrm{La}, \mathrm{Nd}$, and Dy) samples. The figure shows observed intensity $\left(\mathrm{Y}_{\mathrm{obs}}\right)$, calculated intensity $\left(\mathrm{Y}_{\text {calc }}\right)$, and difference in observed and calculated intensities $\left(\mathrm{Y}_{\mathrm{obs}}-\mathrm{Y}_{\text {calc }}\right)$ and peak positions of different phases are shown at the base line as small ticks (|).

the BLFO and BNFO samples are well matching with the earlier reported data [17]. $\mathrm{Nd}$ addition at $\mathrm{Bi}$ site is helpful in suppressing the secondary phase in BFO. Therefore present samples have a single phase triclinic perovskite structure with all constituent components forming a solid solution rather than a mixture of $\mathrm{Bi}_{2} \mathrm{O}_{3}, \mathrm{Fe}_{2} \mathrm{O}_{3}, \mathrm{Nd}_{2} \mathrm{O}_{3}$, or any other impurity phase except $\mathrm{Bi}_{2} \mathrm{Fe}_{4} \mathrm{O}_{9}$ observed in pure BFO.

Similarly, for BDFO compound, the refinement was performed with $P n m a+R 3 c$ structural model. The Rietveld refined XRD pattern of BDFO is shown in Figure 2(c). The dominant contribution is related to orthorhombic phase (Pnma, 80.62\%) with lattice parameters $a=5.4014(5) \AA$, $b=7.7842(4) \AA$, and $c=5.5904(5) \AA$. The other component is related to rhombohedral phase $(R 3 c, 19.38 \%)$. Reflection conditions obtained for Pnma model are found to be almost similar for $P n 2_{1} a$ model except $h=2 n$ for $h 0 l$. The best iteration gives $\chi^{2} \approx 4.32$ for $R 3 c, \chi^{2} \approx 10.08$ for Pnma, and $\chi^{2} \approx 1.84$ for $R 3 c+$ Pnma model attributing to the fact that crystal structure of BDFO compound is characterized by coexistence of two phases with a minimum $\chi^{2}$ value. The obtained result is consistent with the earlier reported work [14]. Rietveld refined structural parameters of the $\mathrm{Bi}_{0.8} R E_{0.2} \mathrm{FeO}_{3}$ (La, Nd and Dy) samples simulated based on the measured XRD patterns are documented in Table 1.

It is well known that ferroic order and spontaneous polarization in BFO mainly result from the $\mathrm{Bi}^{3+}$ stereochemical $6 \mathrm{~s}^{2}$ lone pair electron. Thus it is expected that systematic doping of rare-earth ions will distort the cation spacing between the oxygen octahedra and alter the long-range ferroelectric order. The ferroelectric properties have a close relation with the $\mathrm{Fe}-$ $\mathrm{O}$ bond length. The interatomic bond lengths of all the four samples were calculated by using Bond_Str program and are tabulated in Table 2. In BLFO compound with rhombohedral $(R 3 c)$ crystal structure the octahedra bond environment is composed of three long degenerate $\mathrm{Fe}-\mathrm{O}$ bond lengths and three short degenerate $\mathrm{Fe}-\mathrm{O}$ bond lengths. The $\mathrm{FeO}_{6}$ 
TABLE 1: Rietveld refined structural parameters of the $\mathrm{Bi}_{0.8} R E_{0.2} \mathrm{FeO}_{3}$ samples simulated based on the measured XRD patterns. The error values are presented in the parentheses.

\begin{tabular}{|c|c|c|c|c|c|c|}
\hline Structure & Cell & Atoms & $x$ & $y$ & $z$ & $R$-factors (\%) \\
\hline \multirow{6}{*}{$\begin{array}{l}R 3 c \\
(100 \%)\end{array}$} & \multicolumn{5}{|c|}{$\mathrm{Bi}_{0.8} \mathrm{La}_{0.2} \mathrm{FeO}_{3}$} & \multirow[b]{2}{*}{$R_{\text {Bragg }}=4.89$} \\
\hline & $a=5.5604(5)$ & $\mathrm{Bi} / \mathrm{La}$ & 0.0000 & 0.0000 & 0.2724 & \\
\hline & $b=5.5604$ & $\mathrm{Fe}$ & 0.0000 & 0.0000 & 0.0000 & $R_{p}=5.36$ \\
\hline & $c=13.7596$ & $\mathrm{O}$ & 0.6679 & 0.7647 & 0.5489 & $R_{\mathrm{wp}}=7.15$ \\
\hline & \multirow[t]{2}{*}{$V=368.43$} & & & & & \multirow{2}{*}{$\begin{array}{l}\chi^{2}=2.91 \\
\mathrm{GoF}=1.7\end{array}$} \\
\hline & & & & & & \\
\hline \multirow{6}{*}{$\begin{array}{l}P 1 \\
(100 \%)\end{array}$} & \multicolumn{5}{|c|}{$\mathrm{Bi}_{0.8} \mathrm{Nd}_{0.2} \mathrm{FeO}_{3}$} & \multirow{6}{*}{$\begin{array}{c}R_{\text {Bragg }}=10.0 \\
R_{p}=7.79 \\
R_{\mathrm{wp}}=11.2 \\
\chi^{2}=1.85 \\
\mathrm{GOF}=1.16\end{array}$} \\
\hline & $a=3.9074$ & $\mathrm{Bi} / \mathrm{Nd}$ & 0.0000 & 0.0000 & 0.0000 & \\
\hline & $b=3.9112(6)$ & $\mathrm{Fe}$ & 0.5689 & 0.4362 & 0.5467 & \\
\hline & $c=3.9002(5)$ & $\mathrm{O} 1$ & -0.0815 & 0.4542 & 0.6774 & \\
\hline & \multirow[t]{2}{*}{$V=59.60(2)$} & $\mathrm{O} 2$ & 0.4538 & -0.0722 & 0.6835 & \\
\hline & & $\mathrm{O} 3$ & 0.4541 & 0.4756 & 0.0176 & \\
\hline \multirow{7}{*}{$\begin{array}{l}\text { Pnma } \\
(80.62 \%)\end{array}$} & & & ${ }_{.8} \mathrm{Dy}_{0.2} \mathrm{FeC}$ & & & \multirow{6}{*}{$\begin{array}{c}R_{B 1}=10.7 \\
R_{B 2}=6.96 \\
R_{p}=5.62 \\
R_{\mathrm{wp}}=7.2 \\
\chi^{2}=1.85\end{array}$} \\
\hline & $a=5.4014$ & $\mathrm{Bi} / \mathrm{Dy}$ & 0.0472 & 0.2500 & 0.9933 & \\
\hline & $b=7.7822$ & $\mathrm{Fe}$ & 0.0000 & 0.0000 & 0.5000 & \\
\hline & $c=5.5904(5)$ & $\mathrm{O} 1$ & 0.3832 & 0.2500 & 0.0818 & \\
\hline & $V=234.99$ & $\mathrm{O} 2$ & 0.2076 & 0.5414 & 0.2044 & \\
\hline & & & & & & \\
\hline & $a=5.5504(2)$ & $\mathrm{Bi} / \mathrm{Dy}$ & 0.0000 & 0.0000 & 0.2676 & \multirow[t]{4}{*}{$\mathrm{GOF}=1.4$} \\
\hline \multirow{3}{*}{$\begin{array}{l}R 3 c \\
(19.38 \%)\end{array}$} & $b=5.5504(2)$ & $\mathrm{Fe}$ & 0.0000 & 0.0000 & 0.0000 & \\
\hline & $c=13.7888$ & $\mathrm{O}$ & 0.6794 & 0.7801 & 0.5544 & \\
\hline & $V=367.88$ & & & & & \\
\hline
\end{tabular}

TABLE 2: Important bond lengths of $\mathrm{Bi}_{0.8} R E_{0.2} \mathrm{FeO}_{3}(R E=\mathrm{La}, \mathrm{Nd}$, and Dy) samples. The error values are presented in the parentheses.

\begin{tabular}{lcc}
\hline Compounds & Bond type & Bond length $(\AA)$ \\
\hline \multirow{2}{*}{ BLFO } & Fe-O (3) & 1.7775 \\
& Fe-O (3) & 2.3165 \\
\hline \multirow{3}{*}{ BNFO } & Fe-O (1) & 1.4653 \\
& Fe-O (1) & 1.9015 \\
& Fe-O (1) & 2.0505 \\
& Fe-O (1) & 2.1114 \\
& Fe-O (1) & 2.1248 \\
BDFO & Fe-O (1) & 2.5882 \\
& Fe-O (2) & 1.9760 \\
& Fe-O (2) & 2.0226 \\
& Fe-O (2) & 2.0958 \\
\hline
\end{tabular}

octahedron gets distorted due to $\mathrm{Nd}$ and Dy substitution, resulting in change in bond lengths as mentioned in Table 2.

3.2. Raman Analysis. Raman spectroscopy is a powerful tool to probe the structural and vibrational property of a material and also provide valuable information about ionic substitution and electric polarization. Raman spectra of BLFO, BNFO, and BDFO samples with excitation wavelength of $488 \mathrm{~nm}$ at room temperature are shown in Figure 3.
It has been reported that undoped $\mathrm{BiFeO}_{3}$ with distorted rhombohedral structure, $R 3 c$ space group, and ten atoms in a unit cell of this structure yields 18 optical phonon modes that can be summarized using the following irreducible representation: $\Gamma_{\mathrm{opt}}=4 A_{1}+5 A_{2}+9 E$; according to group theory 13 observed modes $\left(\Gamma_{\text {Raman }, R 3 c}=4 A_{1}+9 E\right)$ are Raman active, whereas $5 A_{2}$ are Raman inactive modes $[22,23]$. The dependence of mode positions on BLFO, BNFO, and BDFO samples is summarized in Table 3 .

In the present study, for BLFO ceramic $A_{1}$ and $E$ symmetry normal modes for $R 3 c$ symmetry including $A_{1}-1$, $A_{1}-2, A_{1}-3, A_{1}-4, E-1, E-4, E-6, E-7, E-8$, and $E-9$ at around $138,174,191,434,67,273,373,475,527$, and $627 \mathrm{~cm}^{-1}$ are clearly observed. These results are matched well with the Raman active vibration modes identified for BFO $(R 3 c)$ [20, $24]$. The ferroelectricity of BFO generally originates from the stereochemical activity of the $\mathrm{Bi}^{3+} 6 \mathrm{~s}^{2}$ lone pair electron that is mainly responsible for the change in both $\mathrm{Bi}-\mathrm{O}$ covalent bonds. The low frequency characteristic modes below $200 \mathrm{~cm}^{-1}$ may be responsible for the ferroelectric nature of the bismuth ferrite samples. As evident from the X-ray diffraction the changes in crystal symmetries are attributed to the $A$-site disorder created by $\mathrm{Nd}$ and Dy substitution, which leads to the shifting of Raman modes with sudden disappearance of some modes. The $A_{1}-2, A_{1}-3$, and $A_{1}-4$ were decomposed completely in $\mathrm{BNFO}$ and $\mathrm{BDFO}$ samples. 
TABle 3: Raman modes $\left(\mathrm{cm}^{-1}\right)$ for $\mathrm{Bi}_{0.8} R E_{0.2} \mathrm{FeO}_{3}\left(R E=\mathrm{La}, \mathrm{Nd}\right.$, and Dy) samples and the bulk $\mathrm{BiFeO}_{3}$ (Kothari et al. [20]).

\begin{tabular}{|c|c|c|c|c|}
\hline Raman modes $\left(\mathrm{cm}^{-1}\right)$ & BFO bulk [20] & BLFO & BNFO & $\mathrm{BDFO}$ \\
\hline$A_{1}-1$ & 135.15 & 135.95 & 139.06 & 142.33 \\
\hline$A_{1}-2$ & 167.08 & 174.52 & - & 176.88 \\
\hline$A_{1}-3$ & 218.11 & - & - & - \\
\hline$A_{1}-4$ & 430.95 & 434.14 & - & - \\
\hline$E-1$ & 71.39 & 67.44 & 68.24 & - \\
\hline$E-2$ & 98.36 & - & 97.91 & - \\
\hline$E-3$ & 255.38 & - & - & 234.32 \\
\hline$E-4$ & 283.0 & 273.93 & 298.01 & 278.77 \\
\hline$E-5$ & 321.47 & - & - & 326.52 \\
\hline$E-6$ & 351.55 & 373.35 & - & 386.31 \\
\hline$E-7$ & 467.60 & 475.53 & 488.53 & 475.47 \\
\hline$E-8$ & 526.22 & 527.78 & - & 531.89 \\
\hline$E-9$ & 598.84 & 627.47 & 619.53 & 585.45 \\
\hline
\end{tabular}
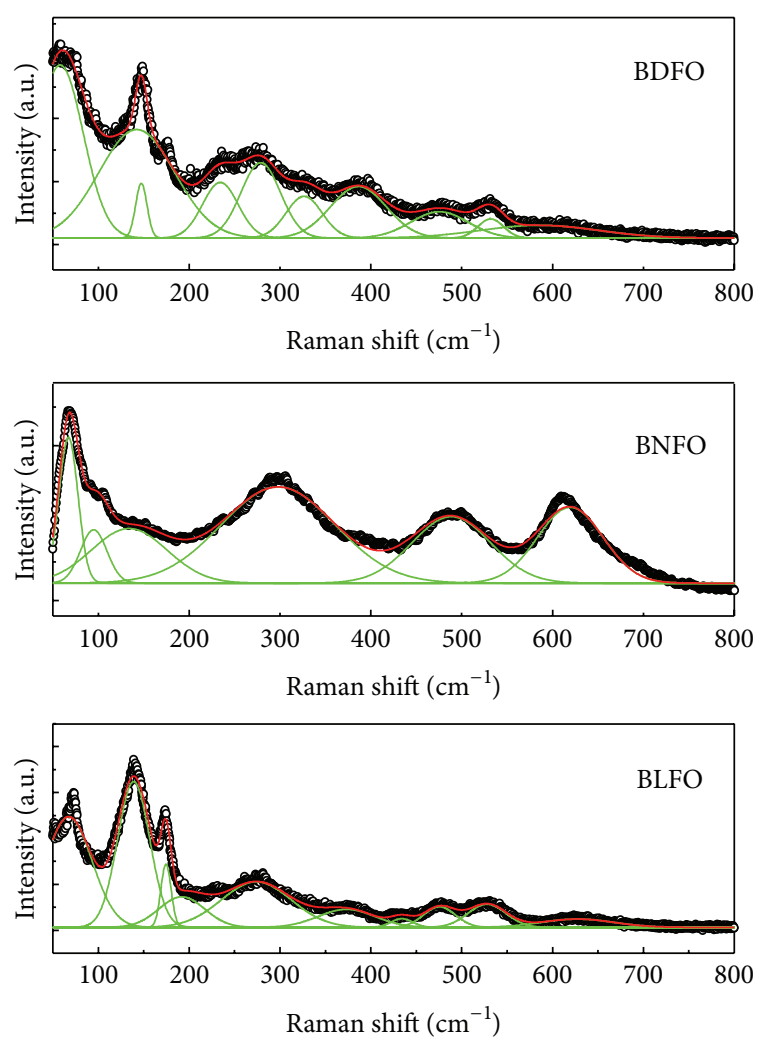

FIGURE 3: Raman spectra for $\mathrm{Bi}_{0.8} R E_{0.2} \mathrm{FeO}_{3}(R E=\mathrm{La}, \mathrm{Nd}$, and $\mathrm{Dy})$ samples at room temperature with excitation wavelength of $488 \mathrm{~nm}$.

The normal modes related to the $\mathrm{Bi}-\mathrm{O}$ covalent bonds (i.e., $E-1, A_{1}-1, A_{1}-2, A_{1}-3$, and $E-2$ modes) shift gradually toward higher frequencies and are attributed to the substitution of relatively light (mass) $\mathrm{La}^{3+}(138.90 \mathrm{~g}), \mathrm{Nd}^{3+}(144.24 \mathrm{~g})$, and $\mathrm{Dy}^{3+}(162.50 \mathrm{~g})$ ion for $\mathrm{Bi}^{3+}(208.98 \mathrm{~g})$ ion in the $\mathrm{BiFeO}_{3}$.

3.3. Ferroelectric (P-E) Measurement. We have also made attempts to measure ferroelectric hysteresis loop at room temperature for $\mathrm{BLFO}, \mathrm{BNFO}$, and $\mathrm{BDFO}$ compounds (Figure 4). It has been observed from the graph that BLFO and BDFO sample represent ferroelectric (FE) behaviour whereas BNFO sample represents paraelectric (PE) nature. The BLFO compound represents ferroelectric loop without any saturation value. This is attributed to the fact that BLFO sample is highly conductive at room temperature which results in the partial reversal of the polarization. For BDFO the spontaneous polarization $\left(2 P_{s}\right)$, remnant polarization $\left(2 P_{r}\right)$, and coercive field $\left(E_{c}\right)$ are about $\sim 0.23 \mu \mathrm{c} / \mathrm{cm}^{2}, 0.16 \mu \mathrm{c} / \mathrm{cm}^{2}$, and $1.50 \mathrm{kV} / \mathrm{cm}$, respectively, under the electric field of $\sim 3 \mathrm{kV} / \mathrm{cm}$, whereas for BNFO compound the obtained values of $2 P_{s}$ and $2 P_{r}$ are found to be $\sim 2.6 \mu \mathrm{c} / \mathrm{cm}^{2}$ and $\sim 0.0 \mu \mathrm{c} / \mathrm{cm}^{2}$, respectively, under the electric field of $\sim 100 \mathrm{kV} / \mathrm{cm}$.

It has been reported that Dy substitution is very much supportive in decreasing the leakage behavior of $\mathrm{BiFeO}_{3}$. Moreover, too much Dy substitution will degrade ferroelectric nature [25]. This is attributed to the fact that higher Dy substitution will transform the crystal structure from rhombohedral to orthorhombic symmetry. The orthorhombic structure is more centrosymmetric, which in turn suppresses the ferroelectricity [26]. Furthermore, the origin of ferroelectricity in $\mathrm{BiFeO}_{3}$ is generally due to $\mathrm{Bi}^{3+}\left(6 \mathrm{~s}^{2}\right)$ lone pair electron. The substitution of $\mathrm{Bi}^{3+}$ with $\mathrm{Dy}^{3+}$ ion will weaken the stereochemical activity of lone pair and weaken the ferroelectricity. In present study, despite $20 \%$ Dy substitution, BDFO compound is ferroelectric in nature with a small leakage current. This might be because of the coexistence of two phases $(P n m a+R 3 c)$ in present BDFO compound.

Furthermore, for paraelectric BNFO sample the obtained values of $2 P_{s}\left(\sim 2.6 \mu \mathrm{c} / \mathrm{cm}^{2}\right)$ and $2 P_{r}\left(\sim 0 \mu \mathrm{c} / \mathrm{cm}^{2}\right)$ are found to be much lower even at a high field of $100 \mathrm{kV} / \mathrm{cm}$. The lower $P_{r}$ value of BNFO despite its higher applied electric field indicates that $\mathrm{Nd}$ doping degrades the ferroelectric nature. In the present case of $\mathrm{BNFO}$, the $\mathrm{Bi}^{3+}$ lone pair electron hybridizes with empty $p$ orbital of $\mathrm{Bi}^{3+}$ or an $\mathrm{O}^{2-}$ ion to form $\mathrm{Bi}-\mathrm{O}$ covalent bonds ensuing the noncentrosymmetric distortion 

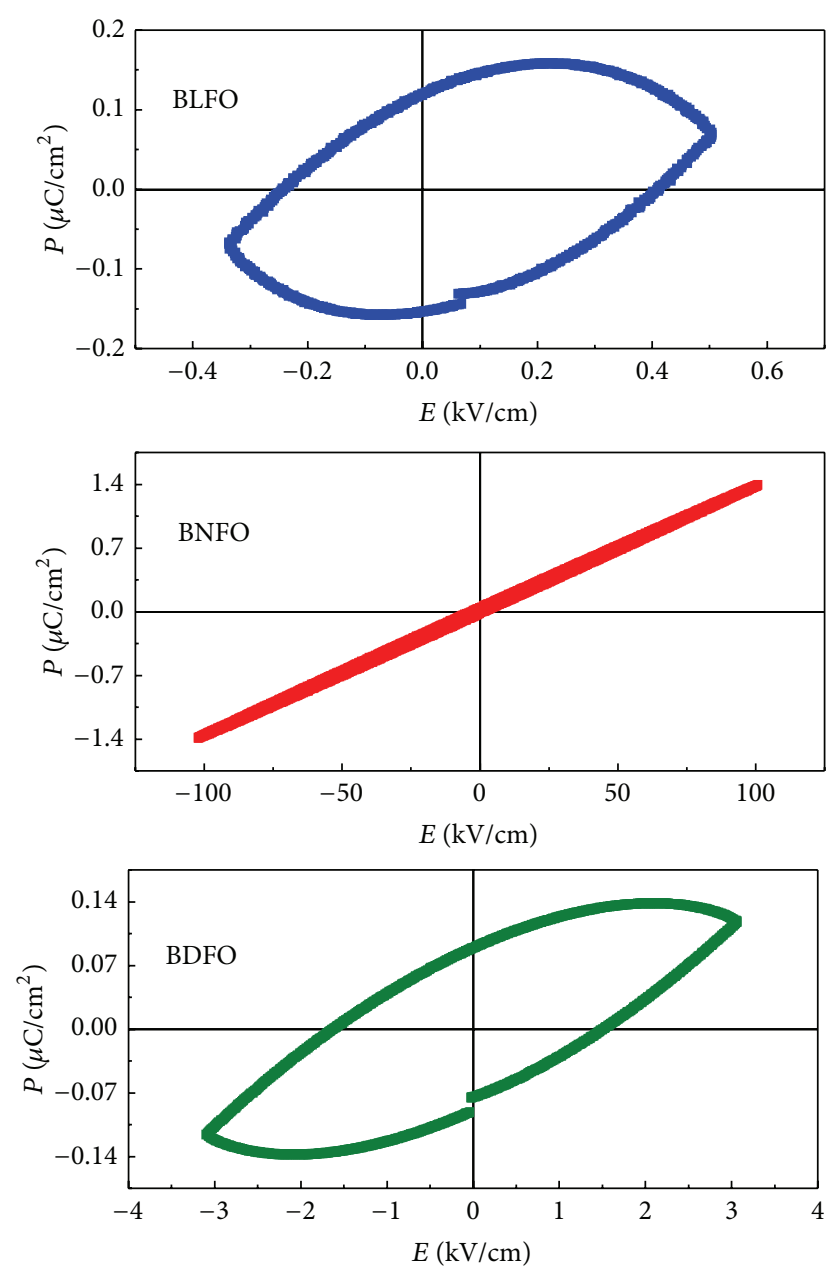

FIgURE 4: Room temperature ferroelectric $(P-E)$ loop measurement of $\mathrm{Bi}_{0.8} R E_{0.2} \mathrm{FeO}_{3}(R E=\mathrm{La}, \mathrm{Nd}$, and $\mathrm{Dy})$ samples.

and ferroelectricity. Substitution of $\mathrm{Nd}^{3+}$ ion weakens the stereochemical activity and results in FE-PE transition.

3.4. Magnetic Hysteresis Analysis. The magnetization-magnetic field $(M-H)$ curve recorded at room temperature for BLFO and BDFO samples with a maximum applied field $10 \mathrm{kOe}$ has been illustrated in Figure 5. Similarly, for BNFO sample, the maximum applied magnetic field $\left(H_{m}\right)$ up to $50 \mathrm{kOe}$ has been also shown in Figure 5. It is well known that pristine BFO has $G$-type antiferromagnetic order with a long period of $\sim 62 \mathrm{~nm}$ of canted spins between two successive ferromagnetically coupled (111) planes and zero net magnetization [27]. There are sharp and noticeable changes in magnetization observed for $\mathrm{Bi}_{0.8} R E_{0.2} \mathrm{FeO}_{3}(R E=\mathrm{Nd}$ and Dy) samples at room temperature. Magnetization increases presentably with doping content as shown in the inset of Figure 5.

In Figure 5(a) $M-H$ curve of BLFO sample shows a very narrow magnetic hysteresis loop, no saturation magnetization, with a small but nonzero remnant magnetization $\left(M_{r}\right)$ of $\sim 0.005 \mathrm{emu} / \mathrm{g}$ and coercive field $\left(H_{c}\right)$ of $\sim 454.6$ Oe. From Figure 5(b), it is clearly seen that BDFO sample exhibits typical ferromagnetic hysteresis loop. The saturation magnetization $\left(M_{s}\right), M_{r}$, and $H_{c}$ values were found to be $1.36 \mathrm{emu} / \mathrm{g}$, $0.05 \mathrm{emu} / \mathrm{g}$, and $4.71 \mathrm{Oe}$, respectively. A weak ferromagnetic behaviour was observed for BLFO and BDFO sample due to the suppression of space modulated spin structure [28, 29]. Doping by $\mathrm{La}$ at $\mathrm{Bi}$ site in BFO leads to distortion in rhombohedral structure. However, doping of Dy further enhances the distortion in rhombohedral structure leading to coexistence of orthorhombic phase (Pnma, 80.62\%) + rhombohedral phase $(R 3 c, 19.38 \%)$. We thus make a note that apart from the suppression of space modulated spin structure mismatch of ionic radii of La and Dy ion leading to different structures is also plausible for weak ferromagnetic behaviour. This can be probed by neutron diffraction and we shall study this problem in near future.

Figure 5(c) represents the room temperature $M-H$ curve for BNFO sample with a maximum applied magnetic field $\left(H_{m}\right) 50 \mathrm{kOe}$. The magnetic field dependent magnetization of BNFO showed similar behaviour. In case of ceramic BNFO, it exhibits a deviation from linear loop at $300 \mathrm{~K}$ as documented in Figure 5(c). Interestingly, the peculiar double hysteresis loop like $M-H$ curve in both BNFO samples with a low coercive field $\left(H_{c}\right)$ has been observed; however this implies that additional factors contribute to enhanced magnetization. Similar behaviour was earlier observed in Ho doped BFO and is attributed to ferromagnetic interaction between $\mathrm{Fe}^{3+}$ and $\mathrm{Ho}^{3+}[15]$.

The magnetization appears in all the samples due to suppression of spiral magnetic spin structures in $\mathrm{Bi}_{0.8} R E_{0.2} \mathrm{FeO}_{3}$ $(R E=\mathrm{La}, \mathrm{Nd}$, and $\mathrm{Dy})$. As the substituting ions effectively disturb the crystal structure differently reasons for each rareearth dopant in this report are different for destruction of spiral magnetic spin structure [29]. In other words, the RE ion as ( $R E=\mathrm{La}, \mathrm{Nd}$, and Dy) doping in BFO can only suppress but cannot destroy the spin cycloid structure completely at $20 \%$ substitution in BFO. Similar behavior is also observed in previously rare-earth doped $\mathrm{BiFeO}_{3}[10-15,30]$. Henceforth, partial substitution should be considered as one of the most effective ways for improving the multiferroic characteristic of antiferromagnetic BFO.

\section{Conclusion}

Conclusively, polycrystalline $\mathrm{Bi}_{0.8} R E_{0.2} \mathrm{FeO}_{3}(R E=\mathrm{La}, \mathrm{Nd}$, and Dy) samples were successfully prepared through solidstate reaction route and were further investigated by XRD, Raman spectroscopy, and ferroelectric and magnetic measurements. All the samples fitted with Rietveld refinement using FullPROF program revealed the existence of rhombohedral structure with space group $R 3 c$ for BLFO $\left(\mathrm{La}^{3+}\right.$ doped $\mathrm{BiFeO}_{3}$ ) and also revealed the substitutional induced structural transformation $R 3 c \rightarrow P 1$ (BNFO) and $R 3 c \rightarrow$ Pnma (BDFO) systems. The changes in crystal structure are attributed to the $A$-site disorder created by $\mathrm{Nd}$ and $\mathrm{Dy}$ substitution, which leads to the shifting of Raman modes with sudden disappearance of some modes as $A_{1}-2, A_{1}-3$, and $A_{1}-4$ decomposed completely in $\mathrm{BNFO}$ and $\mathrm{BDFO}$ samples. 


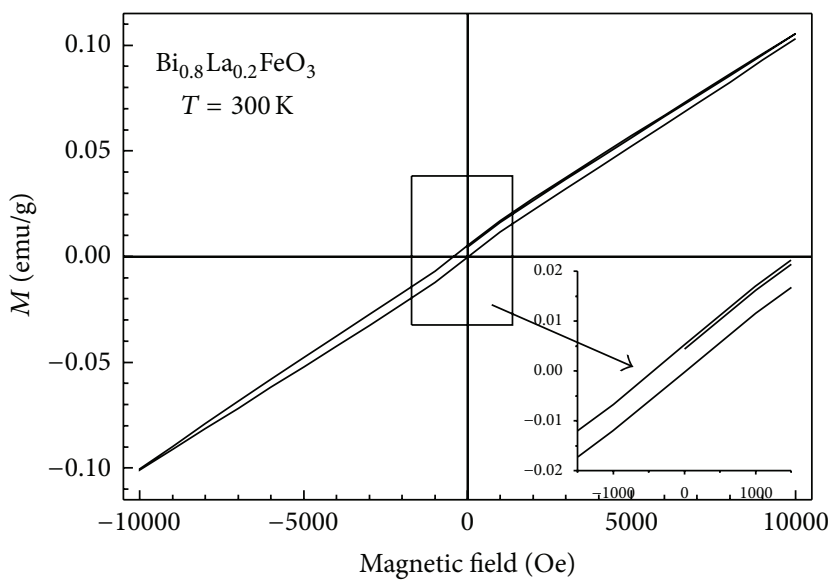

(a)

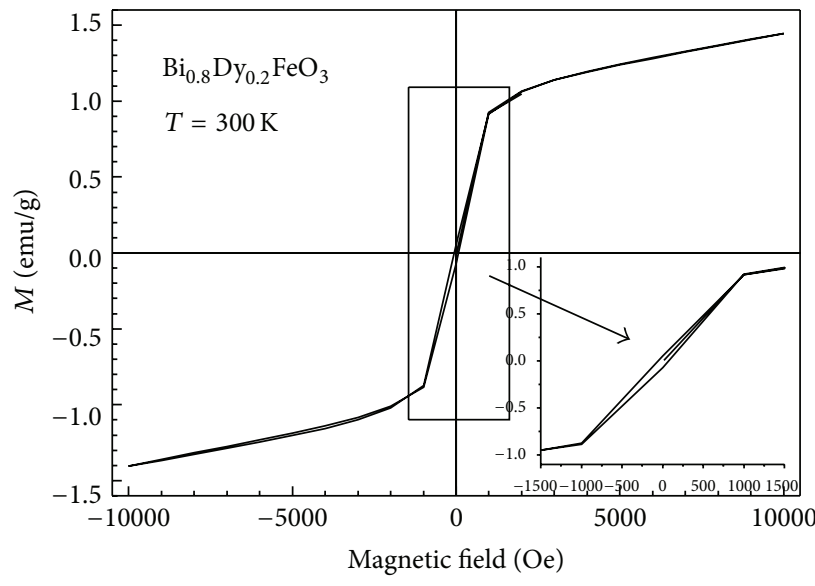

(b)

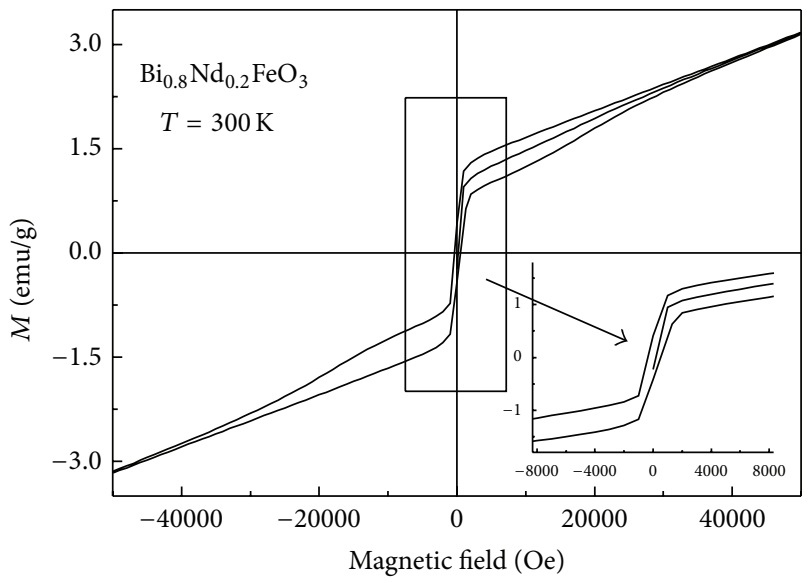

(c)

FIgURE 5: Magnetization versus magnetic field $(M-H)$ loops of $\mathrm{Bi}_{0.8} R E_{0.2} \mathrm{FeO}_{3}(R E=\mathrm{La}, \mathrm{Nd}$, and Dy) samples recorded at room temperature. The inset shows the enlarged data.

Ferroelectric and ferromagnetic loops have been observed in the $\mathrm{Bi}_{0.8} R E_{0.2} \mathrm{FeO}_{3}$ ceramics at room temperature, indicating that ferroelectric and ferromagnetic ordering coexist in the ceramics at room temperature. A significant enhancement in magnetization with enhanced remnant magnetization and coercive field is inferred in rareearth doped $\mathrm{Bi}_{0.8} R E_{0.2} \mathrm{FeO}_{3}(R E=\mathrm{La}, \mathrm{Nd}$, and $\mathrm{Dy})$ samples. These results validate the doping induced destruction of the cycloidal structure in $\mathrm{Bi}_{0.8} R E_{0.2} \mathrm{FeO}_{3}(R E=\mathrm{La}, \mathrm{Nd}$, and $\mathrm{Dy})$ samples.

\section{Conflict of Interests}

The authors declare that there is no conflict of interests regarding the publication of this paper.

\section{Acknowledgments}

Financial support from UGC-DAE Consortium for Scientific Research, Indore, is gratefully acknowledged. XRD, Raman, and $P$-E measurements were performed at UGC-DAE Consortium for Scientific Research, Indore. The authors wish to thank Dr. Mukul Gupta, Dr. V. G. Sathe, and Dr. V. R. Reddy, UGC-DAE CSR, Indore, for useful discussions. They are also grateful to Dr. V. P. S. Awana, National Physical Laboratory, New Delhi, for his timely support in magnetic measurements.

\section{References}

[1] M. Fiebig, "Revival of the magnetoelectric effect," Journal of Physics D: Applied Physics, vol. 38, no. 8, pp. R123-R152, 2005.

[2] G. Catalan and J. F. Scott, "Physics and applications of bismuth ferrite," Advanced Materials, vol. 21, no. 24, pp. 2463-2485, 2009.

[3] N. A. Hill, "Why are there so few magnetic ferroelectrics?" The Journal of Physical Chemistry B, vol. 104, no. 29, pp. 6694-6709, 2000.

[4] D. I. Khomskii, "Multiferroics: different ways to combine magnetism and ferroelectricity," Journal of Magnetism and Magnetic Materials, vol. 306, no. 1, pp. 1-8, 2006.

[5] J. B. Neaton, C. Ederer, U. V. Waghmare, N. A. Spaldin, and K. M. Rabe, "First-principles study of spontaneous polarization in multiferroic $\mathrm{BiFeO}_{3}$," Physical Review B: Condensed Matter and Materials Physics, vol. 71, no. 1, Article ID 014113, 2005. 
[6] I. Sosnowska, T. P. Neumaier, and E. Steichele, "Spiral magnetic ordering in bismuth ferrite," Journal of Physics C: Solid State Physics, vol. 15, no. 23, pp. 4835-4846, 1982.

[7] W. Kaczmarek, Z. Pajas, and M. Połomska, "Differential thermal analysis of phase transitions in $\left(\mathrm{Bi}_{1-x} \mathrm{La}_{x}\right) \mathrm{FeO}_{3}$ solid solution," Solid State Communications, vol. 17, no. 7, pp. 807-810, 1975.

[8] S. Vijayanand, M. B. Mahajan, H. S. Potdar, and P. A. Joy, "Magnetic characteristics of nanocrystalline multiferroic $\mathrm{BiFeO}_{3}$ at low temperatures," Physical Review B-Condensed Matter and Materials Physics, vol. 80, no. 6, Article ID 064423, 2009.

[9] S.-T. Zhang, Y. Zhang, M.-H. Lu et al., "Substitution-induced phase transition and enhanced multiferroic properties of $\mathrm{Bi}_{1-x} \mathrm{La}_{x} \mathrm{FeO}_{3}$ ceramics," Applied Physics Letters, vol. 88, no. 16, Article ID 162901, 3 pages, 2006.

[10] D. Varshney, P. Sharma, S. Satapathy, and P. K. Gupta, "Structural, magnetic and dielectric properties of $\mathrm{Pr}$-modified $\mathrm{BiFeO}_{3}$ multiferroic," Journal of Alloys and Compounds, vol. 584, pp. 232-239, 2014.

[11] G. L. Yuan, S. W. Or, J. M. Liu, and Z. G. Liu, "Structural transformation and ferroelectromagnetic behavior in singlephase $\mathrm{Bi}_{1-x} \mathrm{Nd}_{x} \mathrm{FeO}_{3}$ multiferroic ceramics," Applied Physics Letters, vol. 89, no. 5, Article ID 052905, 2006.

[12] G. L. Yuan and S. W. Or, "Enhanced piezoelectric and pyroelectric effects in single-phase multiferroic $\mathrm{Bi}_{1-x} \mathrm{Nd}_{x} \mathrm{FeO}_{3}(x=0-$ 0.15) ceramics," Applied Physics Letters, vol. 88, p. 062905, 2006.

[13] V. A. Khomchenko, D. A. Kiselev, I. K. Bdikin et al., "Crystal structure and multiferroic properties of Gd-substituted $\mathrm{BiFeO}_{3}$," Applied Physics Letters, vol. 93, no. 26, Article ID 262905, 2008.

[14] V. A. Khomchenko, D. V. Karpinsky, A. L. Kholkin et al., "Rhombohedral-to-orthorhombic transition and multiferroic properties of Dy-substituted $\mathrm{BiFeO}_{3}$," Journal of Applied Physics, vol. 108, no. 7, Article ID 074109, 2010.

[15] N. Jeon, D. Rout, W. Kim, and S. L. Kang, "Enhanced multiferroic properties of single-phase $\mathrm{BiFeO}_{3}$ bulk ceramics by $\mathrm{Ho}$ doping," Applied Physics Letters, vol. 98, Article ID 072901, 2011.

[16] J. Rodríguez-Carvajal, "Recent advances in magnetic structure determination by neutron powder diffraction," Physica B: Physics of Condensed Matter, vol. 192, no. 1-2, pp. 55-69, 1993.

[17] A. Kumar and D. Varshney, "Crystal structure refinement of $\mathrm{Bi}_{1-x} \mathrm{Nd}_{x} \mathrm{FeO}_{3}$ multiferroic by the Rietveld method," Ceramics International, vol. 38, pp. 3935-3942, 2012.

[18] Z. Tao, Y. Huang, and H. J. Seo, "Blue luminescence and structural properties of $\mathrm{Ce}^{3+}$-activated phosphosilicate apatite $\mathrm{Sr}_{5}\left(\mathrm{PO}_{4}\right) 2\left(\mathrm{SiO}_{4}\right)$," Dalton Transactions, vol. 42, no. 6, pp. 21212129, 2013.

[19] B. D. Cullity, Introduction to Magnetic Materials, AddisonWesley, Reading, Mass, USA, 1972.

[20] D. Kothari, V. R. Reddy, V. G. Sathe, A. Gupta, A. Banerjee, and A. M. Awasthi, "Raman scattering study of polycrystalline magnetoelectric $\mathrm{BiFeO}_{3}$," Journal of Magnetism and Magnetic Materials, vol. 320, no. 3-4, pp. 548-552, 2008.

[21] Q.-H. Jiang, C.-W. Nan, and Z.-J. Shen, "Synthesis and properties of multiferroic La-modified $\mathrm{BiFeO}_{3}$ ceramics," Journal of the American Ceramic Society, vol. 89, no. 7, pp. 2123-2127, 2006.

[22] M. K. Singh, H. M. Jang, S. Ryu, and M. H. Jo, "Polarized Raman scattering of multiferroic $\mathrm{BiFeO}_{3}$ epitaxial films with rhombohedral R3c symmetry," Applied Physics Letters, vol. 88, p. $42907,2006$.
[23] R. Haumont, J. Kreisel, P. Bouvier, and F. Hippert, "Phonon anomalies and the ferroelectric phase transition in multiferroic $\mathrm{BiFeO}_{3}$," Physical Review B, vol. 73, no. 13, Article ID 132101, 2006.

[24] J. Wu and J. Wang, " $\mathrm{BiFeO}_{3}$ thin films of ( 1111$)$-orientation deposited on $\mathrm{SrRuO}_{3}$ buffered $\mathrm{Pt} / \mathrm{TiO}_{2} / \mathrm{SiO}_{2} / \mathrm{Si}\left(\begin{array}{lll}1 & 0 & 0\end{array}\right)$ substrates," Acta Materialia, vol. 58, no. 5, pp. 1688-1697, 2010.

[25] S. Zhang, L. Wang, Y. Chen, D. Wang, Y. Yao, and Y. Ma, "Observation of room temperature saturated ferroelectric polarization in Dy substituted $\mathrm{BiFeO}_{3}$ ceramics," Journal of Applied Physics, vol. 111, no. 7, Article ID 074105, 5 pages, 2012.

[26] W.-M. Zhu, L. W. Su, Z.-G. Ye, and W. Ren, "Enhanced magnetization and polarization in chemically modified multiferroic $(1-x) \mathrm{BiFeO}_{3-x} \mathrm{DyFeO}_{3}$ solid solution," Applied Physics Letters, vol. 94, no. 14, Article ID 142908, 2009.

[27] M. M. Kumar, S. Srunath, G. S. Kumar, and S. V. Suryanarayana, "Spontaneous magnetic moment in $\mathrm{BiFeO}_{3}-\mathrm{BaTiO}_{3}$ solid solutions at low temperatures," Journal of Magnetism and Magnetic Materials, vol. 188, no. 1-2, pp. 203-212, 1998.

[28] D. Maurya, H. Thota, A. Garg, B. Pandey, P. Chand, and H. C. Verma, "Magnetic studies of multiferroic Bil-xSmxFeO ${ }_{3}$ ceramics synthesized by mechanical activation assisted processes," Journal of Physics Condensed Matter, vol. 21, no. 2, Article ID 026007, 2009.

[29] V. A. Khomchenko, D. A. Kiselev, J. M. Vieira et al., "Effect of diamagnetic $\mathrm{Ca}, \mathrm{Sr}, \mathrm{Pb}$, and $\mathrm{Ba}$ substitution on the crystal structure and multiferroic properties of the $\mathrm{BiFeO}_{3}$ perovskite," Journal of Applied Physics, vol. 103, no. 2, Article ID 024105, 2008.

[30] Y.-J. Zhang, H.-G. Zhang, J.-H. Yin et al., "Structural and magnetic properties in $\mathrm{Bi}_{1-x} \mathrm{R}_{x} \mathrm{FeO}_{3}(x=0-1, R=\mathrm{La}, \mathrm{Nd}, \mathrm{Sm}$, $\mathrm{Eu}$ and $\mathrm{Tb}$ ) polycrystalline ceramics," Journal of Magnetism and Magnetic Materials, vol. 322, no. 15, pp. 2251-2255, 2010. 

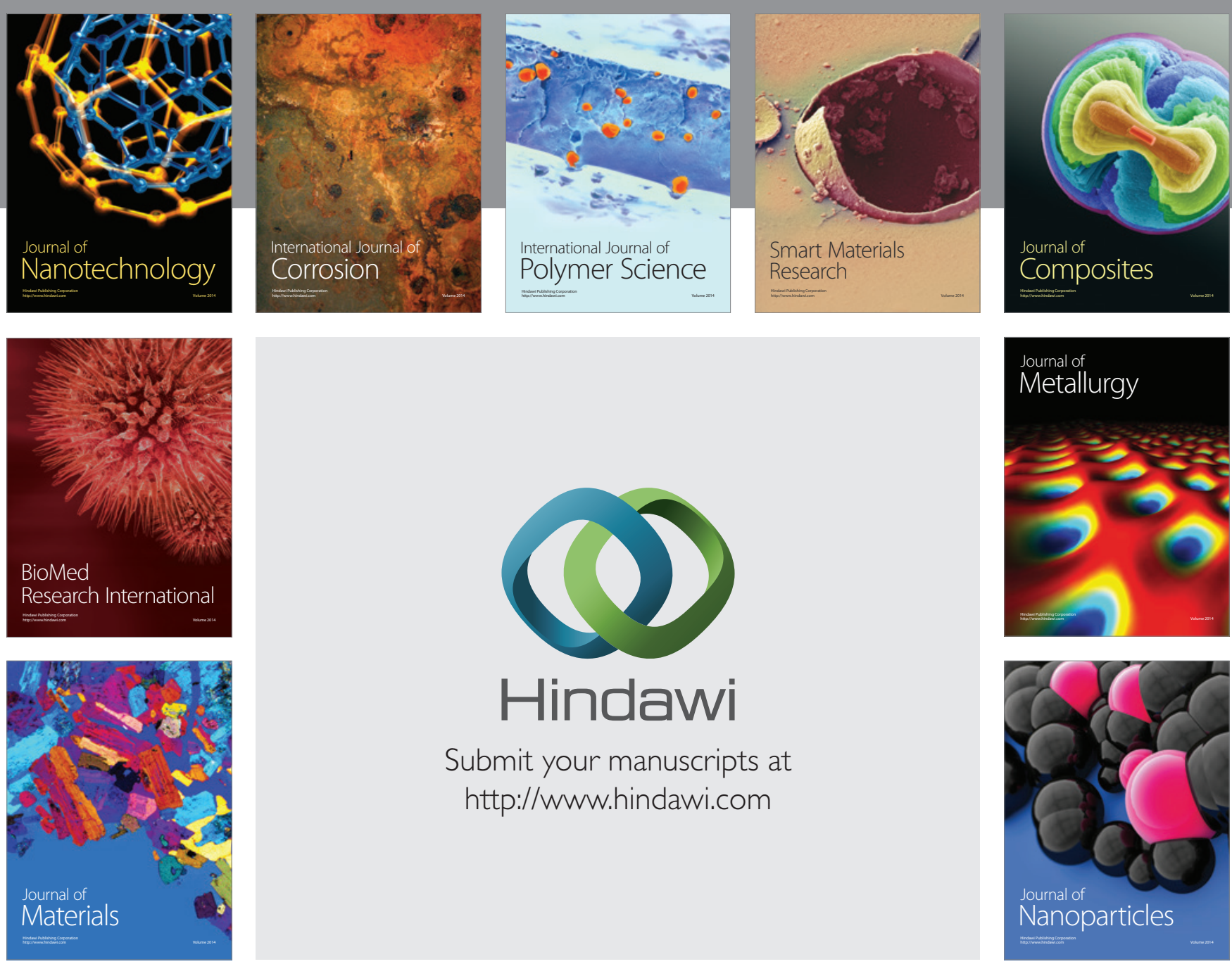

Submit your manuscripts at http://www.hindawi.com
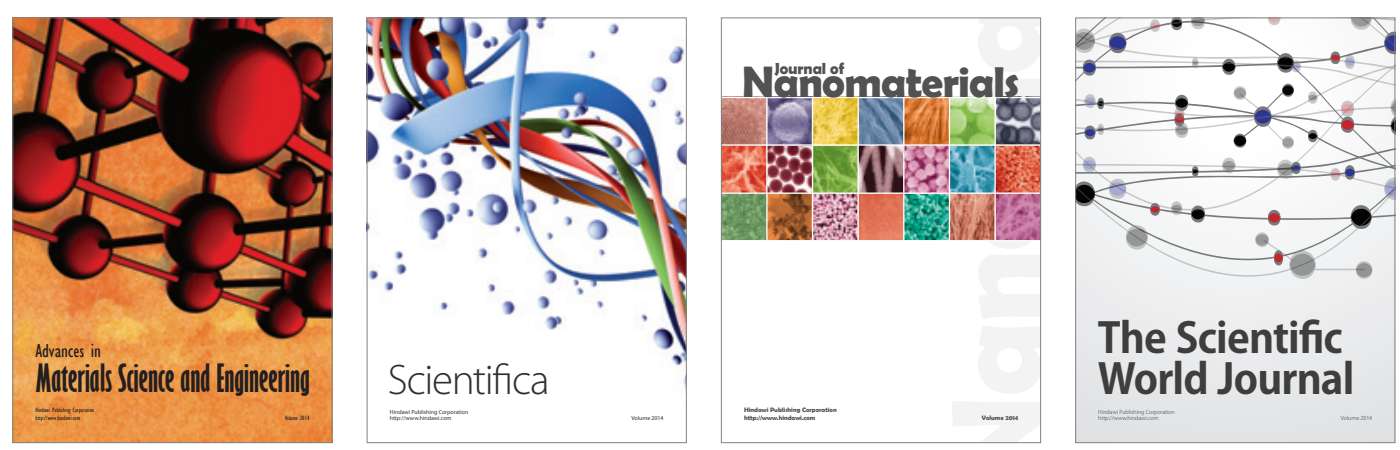

\section{The Scientific World Journal}
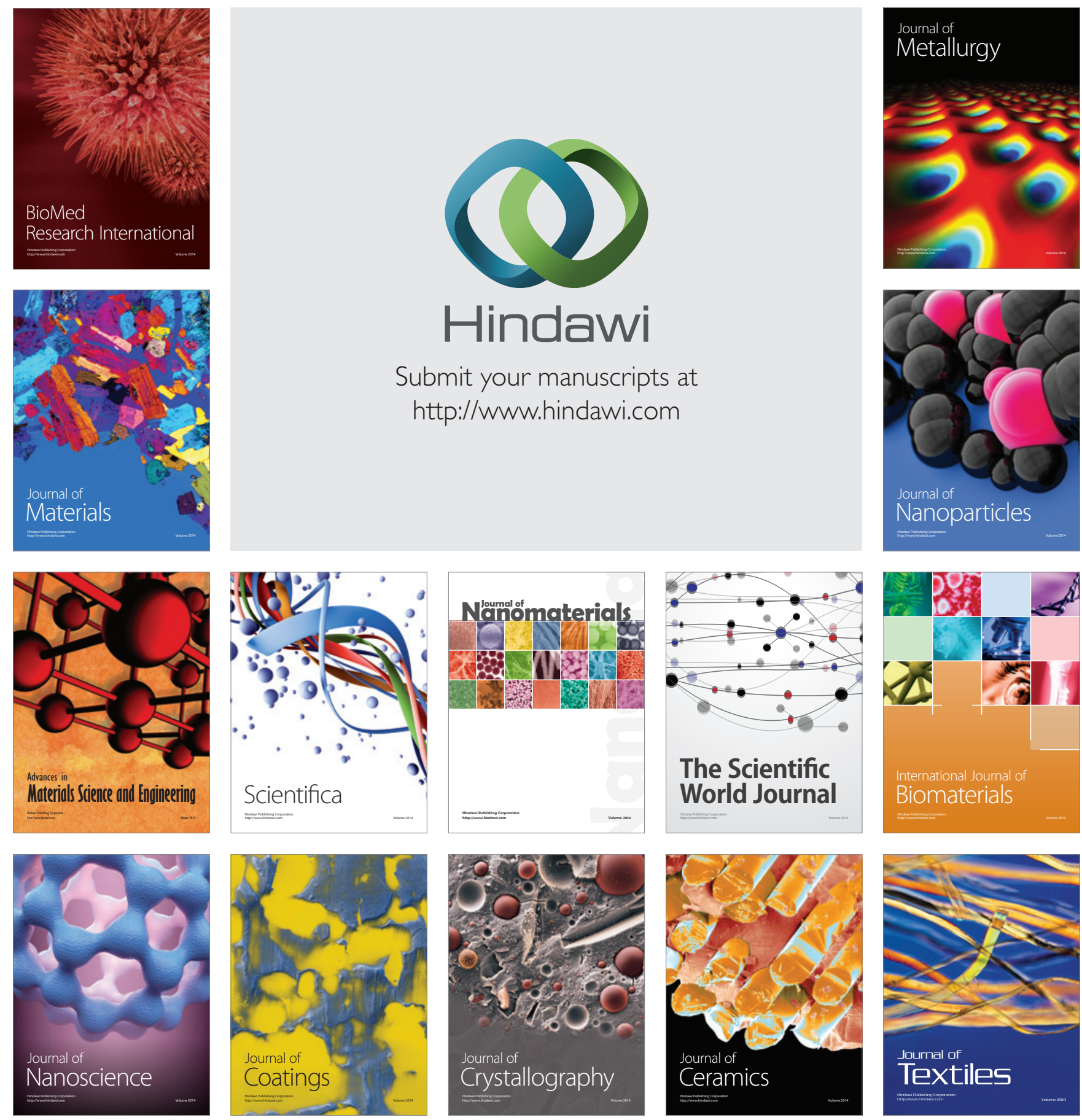